A $\mathrm{Gublications}$ J. Chem. Metrol. 11:2(2017) 40-45

journal of chemical metrology

\title{
Proficiency testing performance of Turkish laboratories on determination of relative composition of fatty acids in sunflower oil
}

\author{
Hasibe Yilmaz $^{1^{*}}$, Simay Gunduz ${ }^{1}$ and Fatma Akcadag ${ }^{\circ}$ \\ ${ }^{1}$ Organic Chemistry Laboratory, TÜBİTAK UME (National Metrology Institute), \\ Gebze, Türkiye \\ ${ }^{2}$ Reference Materials Laboratory, TÜBİTAK UME (National Metrology Institute), \\ Gebze, Türkiye
}

(Received May 25, 2017; Revised July 05, 2017; Accepted July 09, 2017)

\begin{abstract}
Proficiency testing of laboratories in Turkey to compare and evaluate their performances on analysis of fatty acids in sunflower oil was performed. Analysis of the quality control test material allows the laboratory to compare the analytical results with other laboratories. In order to increase the quality of chemical analysis and awareness of metrology, TÜBITTAK UME have been organizing Proficiency Testing Schemes in the field of determination of fatty acids in sunflower oil since 2005. In this study, an overview of the proficiency test for the determination of fatty acids in sunflower oil in Turkey is presented.
\end{abstract}

Keywords: Fatty acids; sunflower oil; GC-MS. C 2017 ACG Publications. All rights reserved.

\section{Introduction}

Sunflower oil is an important energy source for human body, therefore it is important to determine its quality. The quality of sunflower oil is related to the composition and distribution of fatty acids in it. Thus, metrologically, it is important to quantify the amount of fatty acids in sunflower oil [1].

Validated measurement methods are essential for quality assurance efforts. Food Quality Control Laboratories might be accredited according to ISO/IEC 17025 for their quality control/assurance system. Availability of appropriate quality assurance systems are important tools for a good laboratory practice and may include participation in proficiency testing (PT) scheme, which are attended by several testing laboratories to evaluate the performance of their analysis. To assess the analytical capabilities of the laboratories, proficiency testing is important. Analyses of the proficiency test materials allow a laboratory to compare their analytical results with other laboratories [2-8].

In this study, a proficiency testing scheme, had been organised by TÜBITAK UME, and to the participating laboratories homogeneous oil samples were dispached to conduct the analysis and submit their results (the fatty acid composition of sunflower oil; palmitic acid, linoleic acid, stearic acid and oleic acid) to TÜBİTAK UME. The results were evaluated statistically, and the distribution of fatty acid composition in sunflower oil was determined. The composition of fatty acids (as methyl \footnotetext{
(262) 6795001

* Corresponding author: E-Mail: hasibe.yilmaz@tubitak.gov.tr ; Phone: + 90 (262) 6795000 /6517; Fax: + 90 
ester derivatives) in sunflower oil was also investigated in TÜBİTAK UME laboratories by gas chromatography-mass spectrometry (GC-MS) [9,10].

\section{Experimental}

\subsection{Materials}

Methanol $\geq 99.9 \%$, n-hexane $\geq 98.0 \%$, dichloromethane $\geq 99 \%$, sodium chloride $99.5 \%$ and sulfuric acid (95-98) \% were obtained from Merck; sunflower oil was obtained from the market.

\subsection{Method and Instrumentation}

GC-MS analyses were performed using a Thermo Scientific TSQ Quantum XLS GC-MS/MS instrument equipped with a DB-5 column $(30 \mathrm{~m} \times 0.25 \mathrm{mmID} \times 0.25 \mu \mathrm{m})$. Helium was used as a carrier gas at a constant flow rate of $1 \mathrm{~mL} \mathrm{~min}^{-1} .1 \mu \mathrm{L}$ of sample was injected. The GC temperature program was set as follows; $120{ }^{\circ} \mathrm{C}$ hold for $0.50 \mathrm{~min}$, ramp to $220{ }^{\circ} \mathrm{C}$ at $30{ }^{\circ} \mathrm{C} \mathrm{min}{ }^{-1}$ and hold for 1 min then to $240{ }^{\circ} \mathrm{C}$ at $10{ }^{\circ} \mathrm{C} \mathrm{min}{ }^{-1}$ and hold for $5 \mathrm{~min}$. The mass spectrometer was operated in positive EI mode with an ionization energy of $70 \mathrm{eV}$, and collision energy of $5 \mathrm{~V}$. The ion source temperature was $200{ }^{\circ} \mathrm{C}$. The temperature of the MS transfer line was set at $250{ }^{\circ} \mathrm{C}$, using scan mode of a mass range from 50 to $650 \mathrm{~m} / \mathrm{z}$. The components were identified by comparison of their mass spectra with NIST Mass Spectral Library.

Table 1. Determined fatty acid methyl esters.

\begin{tabular}{lcc}
\hline FAME & Molecular formula & Symbol \\
\hline Methyl palmitate & $\mathrm{C}_{17} \mathrm{H}_{34} \mathrm{O}_{2}$ & $\mathrm{C} 16: 0$ \\
Methyl stearate & $\mathrm{C}_{19} \mathrm{H}_{38} \mathrm{O}_{2}$ & $\mathrm{C} 18: 0$ \\
Methyl oleate & $\mathrm{C}_{19} \mathrm{H}_{36} \mathrm{O}_{2}$ & $\mathrm{C} 18: 1$ \\
Methyl linoleate & $\mathrm{C}_{19} \mathrm{H}_{34} \mathrm{O}_{2}$ & $\mathrm{C} 18: 2$ \\
\hline
\end{tabular}

\subsection{Synthesis of fatty acid methyl esters}

$25 \mathrm{mg}$ of oil sample was dissolved in $0.5 \mathrm{~mL}$ of toluene and $1 \mathrm{~mL}$ of $\mathrm{H}_{2} \mathrm{SO}_{4}(1 \%$ in methanol $)$ was then added. The mixture was stirred at $50{ }^{\circ} \mathrm{C}$ for overnight. $2 \mathrm{~mL}$ of aqueous sodium chloride $(5 \%)$ was added and the mixture was extracted with $2 \times 5 \mathrm{~mL}$ of hexane. The organic layer was washed with $2 \mathrm{~mL}$ aqueous solution of $\mathrm{NaHCO}_{3}(2 \%)$, dried over $\mathrm{Na}_{2} \mathrm{SO}_{4}$ and the solvent was evaporated under reduced pressure. The residue was a mixture of pure fatty acid methyl esters.

\subsection{The proficiency testing protocol}

The PT protocol consists of the information of sample preparation, sample distribution, sample storage, the programme of the study and parameters (fatty acids) to determine in sunflower oil. It also provides information on the method of participation, the start and end dates of the proficiency test plan, the dates of the tests to be conducted by the participants, the report of the methods and results, the evaluation of the results, safety precautions and references. The prepared protocol was published on TÜBİTAK UME website and sent to the participants by e-mail. Different laboratory numbers were assigned to each participating laboratory to follow the proficiency test results. In the proficiency testing schemes, privacy of the results and information of the laboratories were considered.

Participants were free to choose the method for the analysis and reported their results online before the submission deadline. All the participants were advised to use their routine procedure in order to compare the quality of their routine measurements.

\subsection{Preparation of PT samples}

Sunflower oils selected as PT samples were obtained from the market. Dry and clean sample bottles were filled with $100 \mathrm{~mL}$ homogeneous oil samples and sent to the participant laboratories. The homogeneity and stability tests were performed by TÜBİTAK UME. 


\section{Results and discussion}

The results of the participants were evaluated statistically and $z$ scores were calculated for each laboratory. The report for proficiency testing was prepared and published on website and the attendance certificates were sent to the participants.

The $\mathrm{z}$ scores were calculated by using the following formula $[1,2]$ :

where,

$$
z_{i}=\frac{x_{i}-x_{p t}}{\sigma_{p t}}
$$

$x_{p t}$ : assigned value,

$x_{i}$ : participant's result

$\sigma_{\mathrm{pt}}:$ standard deviation for proficiency assessment

Interpretation of $z$ scores is as follows [3]:

- $|z| \leq 2.0$ is indicates "satisfactory" performance and generates no signal.

- $2.0<|z|<3.0$ indicates "questionable" performance and generates a warning signal.

- $|z| \geq 3.0$ indicates "unsatisfactory" performance and generates an action signal.

Table 2. The reported data* of participant laboratories in 2008.

\begin{tabular}{|c|c|c|c|c|c|}
\hline Participant & Method & Linoleic Acid & Oleic Acid & Palmitic Acid & Stearic Acid \\
\hline 1 & $\begin{array}{c}\text { TS } 4664 \text { EN ISO } 5508 \text { - } \\
\text { 1996-GC-FID }\end{array}$ & $59.19 \pm 0.056$ & $29.53 \pm 0.03$ & $6.21 \pm 0.02$ & $3.52 \pm 0.035$ \\
\hline 2 & AOAC 963.22-GC-FID & $57.41 \pm 0.01$ & $29.53 \pm 0.054$ & $6.49 \pm 0.186$ & $3.68 \pm 0.01$ \\
\hline 3 & $\begin{array}{c}\text { AOAC-UPAC Method } \\
\text { 969.33, AOAC 2000- } \\
\text { GC-FID }\end{array}$ & $57.66 \pm 0.08$ & $30.46 \pm 0.01$ & $6.43 \pm 0.01$ & $3.62 \pm 0.03$ \\
\hline 4 & $\begin{array}{c}\text { TS } 4664 \text { EN ISO } 5508 \text { - } \\
\text { 1996-GC-FID }\end{array}$ & $58.051 \pm 0.039$ & $30.457 \pm 0.16$ & $6.328 \pm 0.01$ & $3.54 \pm 0.003$ \\
\hline 5 & $\begin{array}{c}\text { TS } 4664 \text { EN ISO } 5508 \text { - } \\
\text { 1996-GC-FID }\end{array}$ & $57.11 \pm 0.01$ & $30.87 \pm 0.01$ & $6.74 \pm 0.01$ & $3.8 \pm 0.01$ \\
\hline 6 & $\begin{array}{c}\text { TS } 4664 \text { EN ISO } 5508 \text { - } \\
\text { 1996-GC-MS }\end{array}$ & $62.07 \pm 0.27$ & $27.27 \pm 0.16$ & $6.79 \pm 0.04$ & $3.86 \pm 0.04$ \\
\hline 7 & TS 4504/TS 4664-GC-FID & $57.36 \pm 0.08$ & $29.79 \pm 0.01$ & $6.46 \pm 0.01$ & $3.64 \pm 0.007$ \\
\hline $\begin{array}{l}\text { TÜBİTAK } \\
\text { UME }\end{array}$ & GC-MS & $57.04 \pm 0.08$ & $30.58 \pm 0.01$ & $6.46 \pm 0.01$ & $3.8 \pm 0.02$ \\
\hline
\end{tabular}

*The standard deviations were used as expanded uncertainties.

TUBITAK UME has been organising proficiency testing scheme for fatty acid composition of sunflower oil since 2005, every year. In this study, the data evaluated in 2008 and 2016 are selected for comparison purpose. The results were reported in relative percentage values by the participant laboratories. The participant laboratories' methods and the reported data are given in Table 2 (2008) and Table 4 (2016). The z-scores from the participant laboratories are shown in Table 3 (2008) and Table 5 (2016). The number of participants according to years are shown in Figure 1. The distribution of the participants' results of linoleic, oleic, palmitic and stearic acid in the proficiency tests are given in Figures 2-5, in which black and blue lines represent z scores between (2) - (-2) and (3) - (-3), respectively. 
Table 3. Distribution of all results (2008)

Linoleic Acid Oleic Acid Palmitic Acid Stearic Acid

\begin{tabular}{lcccc}
\hline Participant number (n) & 8 & 8 & 8 & 8 \\
Average value (\%) & 58.24 & 29.81 & 6.49 & 3.68 \\
Median (\%) & 57.54 & 30.12 & 6.46 & 3.66 \\
Reference value (\%) & 57.67 & 30.72 & 6.44 & 3.15 \\
Standard deviation & 0.101 & 0.101 & 0.064 & 0.023 \\
Maximum value (\%) & 62.07 & 30.87 & 6.79 & 3.86 \\
Minimum value (\%) & 57.04 & 27.27 & 6.21 & 3.52 \\
Distribution range (Max-Min) \% & 2.06 & 1.27 & 1.78 & 0.37 \\
TÜBİTAK-UME (\%) & 57.04 & 30.58 & 6.46 & 3.8 \\
\hline
\end{tabular}

Table 4. The reported data of participant laboratories in 2016

\begin{tabular}{|c|c|c|c|c|c|}
\hline Participant & Method & Linoleic Acid & Oleic Acid & Palmitic Acid & Stearic Acid \\
\hline 1 & TS EN ISO 12966-1 & 59.12 & 29.35 & 5.49 & 3.82 \\
\hline 2 & TGK 2014/53 & 58.98 & 29.73 & 6.76 & 3.75 \\
\hline 3 & TGK 2014/53 & 60.5 & 28.4 & 6.87 & 3.58 \\
\hline 4 & $\begin{array}{c}\text { TS EN ISO 12966-1:2015 TS } \\
\text { EN ISO 12966-2 } 2011\end{array}$ & 59.1 & 29.71 & 6.85 & 3.71 \\
\hline 5 & $\begin{array}{l}\text { TS EN ISO 12966-2, } \\
\text { TS EN ISO 12966-4 }\end{array}$ & 57.96 & 29.33 & 6.79 & 3.98 \\
\hline 6 & TGK 2014/53 & 60.96 & 28.1 & 6.1 & 3.27 \\
\hline UME & GC-MS & $58.7 \pm 0.5$ & $29.34 \pm 0.22$ & $6.81 \pm 0.02$ & $3.84 \pm 0.05$ \\
\hline
\end{tabular}

Table 5. Distribution of all results (2016)

\begin{tabular}{lcccc}
\hline & Linoleic Acid & Oleic Acid & Palmitic Acid & Stearic Acid \\
\hline Participant number (n) & 7 & 7 & 7 & 7 \\
Average value (\%) & 59.33 & 29.14 & 6.52 & 3.71 \\
Median (\%) & 59.10 & 29.34 & 6.79 & 3.75 \\
Reference value (\%) & 58.70 & 29.34 & 6.81 & 3.84 \\
Standard deviation & 4.40 & 2.20 & 0.51 & 0.29 \\
Maximum value (\%) & 60.96 & 29.73 & 6.87 & 3.98 \\
Minimum value (\%) & 57.96 & 28.10 & 5.49 & 3.27 \\
Distribution range (Max-Min) \% & 3.00 & 1.63 & 1.38 & 0.71 \\
TÜBİTAK-UME (\%) & 58.70 & 29.34 & 6.81 & 3.84 \\
\hline
\end{tabular}

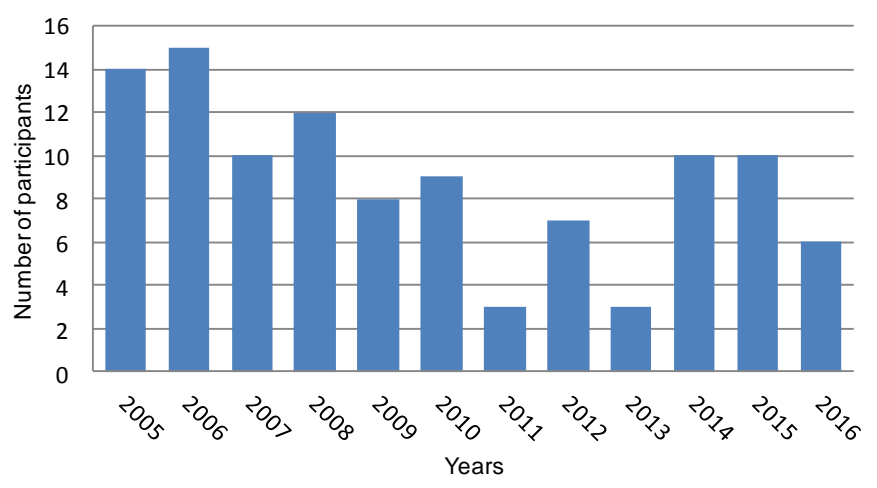

Figure 1. Number of participants by years 


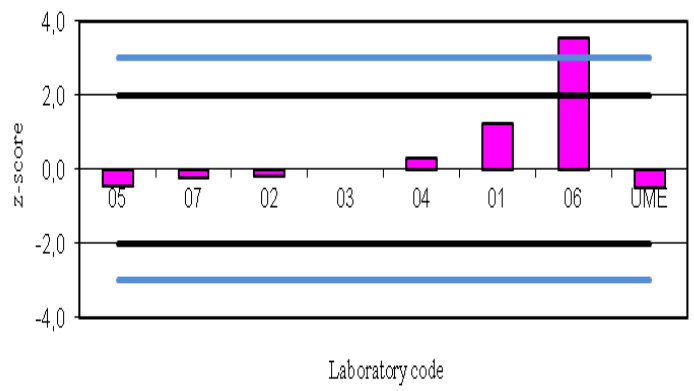

(a)

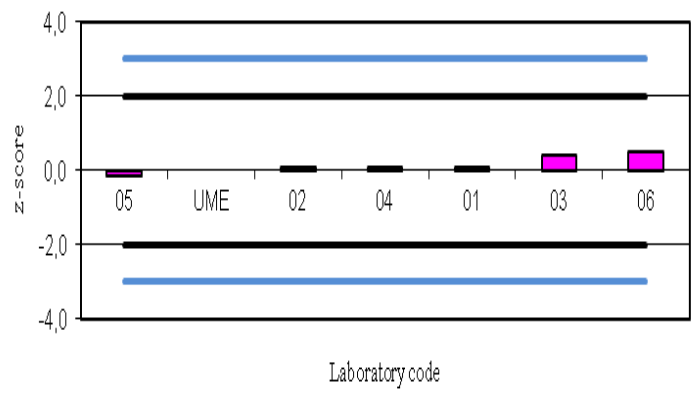

(b)

Figure 2. z-Scores for linoleic acid results in sunflower oil in 2008(a)- 2016(b) PT scheme

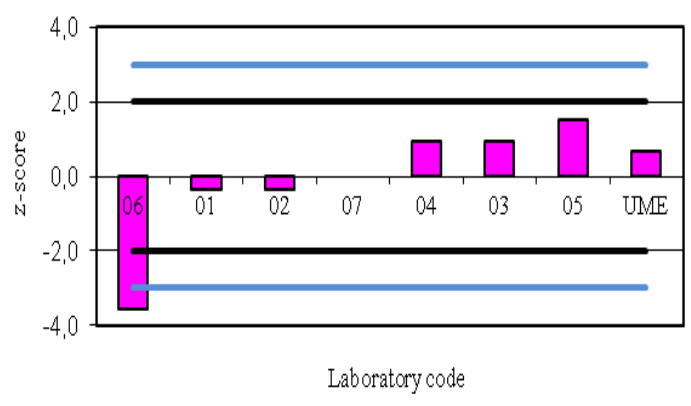

(a)

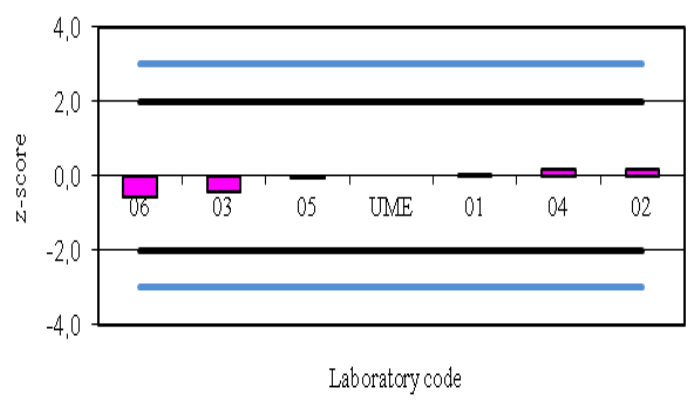

(b)

Figure 3. z-Scores for oleic acid results in sunflower oil in 2008(a)- 2016(b) PT scheme

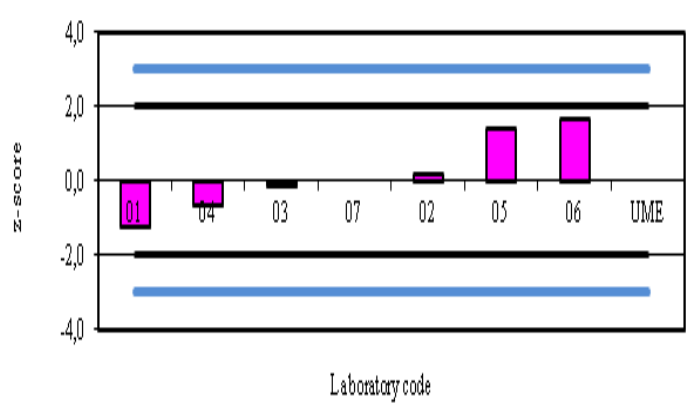

(a)

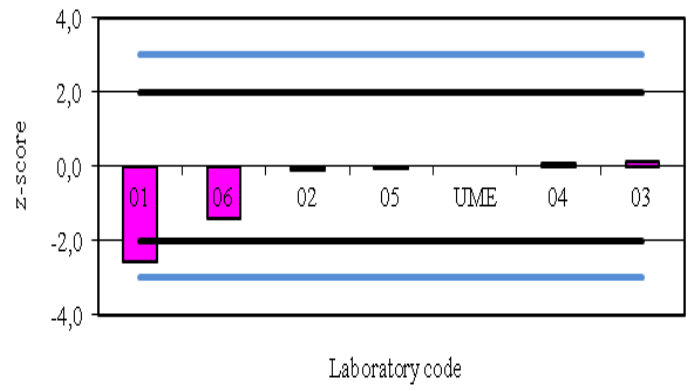

(b)

Figure 4. z-Scores for palmitic acid results in sunflower oil in 2008(a)-2016(b) PT scheme

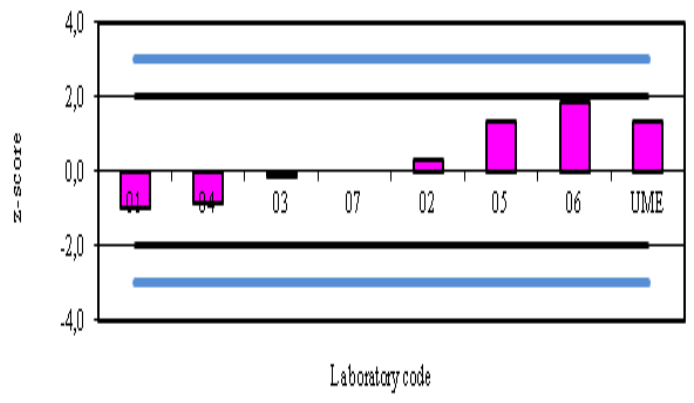

(a)

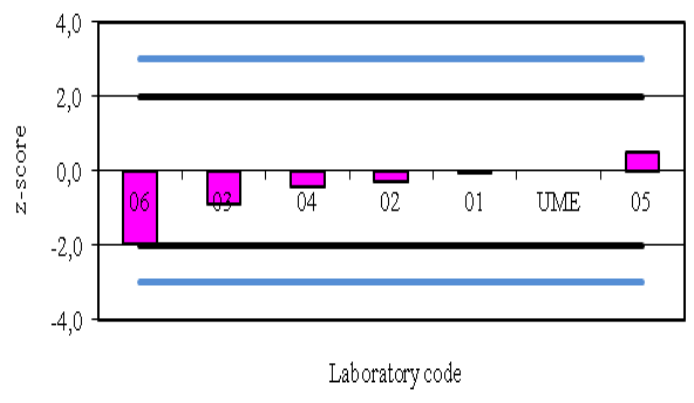

(b)

Figure 5. z-Scores for stearic acid results in sunflower oil in 2008(a)-2016(b) PT scheme 


\section{Conclusion}

In this study, proficiency test results for the fatty acid composition of sunflower oils determined by the participant laboratories over a period of years are evaluated. The results were evaluated statistically and $\mathrm{z}$ scores were reported by TÜBITAK UME. The performances of the laboratories were monitored over a period of time (between 2005 and 2016) and the distribution of the results shows trends in performance of the laboratories. For optimum benefit, continous participation to proficiency testing is important for sustainable laboratory performance.

\section{ORCID}

Hasibe Y1lmaz: 0000-0002-1308-1650

Simay Gündüz: 0000-0003-2243-0098

Fatma Akçadağ: 0000-0003-4000-3874

\section{References}

[1] T.S. Laakso, I. Laakso and R. Hiltunen (2002). Analysis of fatty acids by gas chromatography, and its relevance to research on health and nutrition, Anal. Chim. Act., 465, 39-62.

[2] T. Farrant (1997). Practical statistics for the analytical scientist, Royal Society Chemistry.

[3] ISO 13528. (2015). Statistical methods for use in proficiency testing by interlaboratory comparisons.

[4] ISO/IEC 17043 (2010). Conformity assessment - General requirements for proficiency testing.

[5] ISO/IEC 17025 (2005). General requirements for the competence of testing and calibration laboratories.

[6] R.E. Lawn, M. Thompson and F. R. Walker (1997). Proficiency testing in analytical chemistry, Royal Society Chemistry.

[7] Proficiency Testing as a Tool for Accreditation Bodies and Customers of Laboratories. A report on the feasibility of using PT as a tool to provide third parties with information on laboratory competence (1999). LCG.

[8] Türk Gıda Kodeksi zeytinyaği ve pirina yaği numune alma ve analiz metotları tebliği (tebliğ no: 2010/36), Resmi Gazete, Say1 27665, 07.08.2010.

[9] V. Morales, A.C. Gören, A. Held, M. Bilsel, S. Gündüz and H. Yılmaz (2015). Validation of a GC-IDMS method for the metrologically traceable quantification of selected FAMEs in biodiesel, Accred. Qual. Assur. 20, 411-419.

[10] M. Petrovic, N. Kezic and V. Bolanca (2010). Optimization of the GC method for routine analysis of the fatty acid profile in several food samples, Food Chemistry, 122, 285-291.

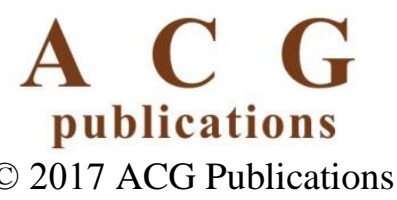

